\title{
The Expansion of Human Body Boundary Explored by Wearable Devices such as Smart Bracelets from the Perspective of Phenomenology
}

\section{Li Yiwen}

School of Humanities and Communication, Zhejiang Gongshang University, Hangzhou, China

\section{Email address:}

18057321036@163.com

To cite this article:

Li Yiwen. The Expansion of Human Body Boundary Explored by Wearable Devices such as Smart Bracelets from the Perspective of Phenomenology. Science Innovation. Vol. 9, No. 4, 2021, pp. 129-133. doi: 10.11648/j.si.20210904.12

Received: April 19, 2021; Accepted: May 17, 2021; Published: May 24, 2021

\begin{abstract}
In recent years, with the rapid development and innovation of technology, intelligent media has gradually entered our daily life, silently changing our way of life. As one of the authorized forms of intelligent technology in the media, intelligent media not only changes our way of life, but also has an impact on the exploration of the sense of boundary in people's self-cognition. In particular, the embedding of wearable devices has triggered more thinking about anthropic questions like, where is the boundary between human and machine, and what is the real existence of human under virtual reality technology. However, the current academic research on wearable devices focuses on technical materials and practical applications, the research of the concept of it with the human body itself is very scarce, relevant empirical research is not enough. In recent years, research in the field of physical transmission has increased gradually, discussion on the body of these theses rise slowly, need to give more attention to the development. In combination with Merleau Ponty's body phenomenology, this paper considers the impact of wearable devices such as smart bracelet on perceptive body, human self-cognition and the boundary relationship between human body and machine from the perspective of phenomenology, and discusses the exploration and extension of wearable devices on human body's sense of boundary from the scale of human value.
\end{abstract}

Keywords: Smart Media, Wearable Devices, Smart Bracelet, Merleau Ponty

\section{现象学视域下智能手环等可穿戴设备对人体边界的探索延伸}

\section{李神雯}

浙江工商大学人文与传播学院, 杭州, 中国

\section{邮箱}

18057321036@163.com

摘要: 近年来, 随着技术日新月异的不断发展革新, 智能媒介逐渐进入到我们的日常生之中, 无声无息地改变着我们 的生活方式。作为智能技术在媒介上的附权形式之一，智能媒介，不仅改变我们的生活方式，而且对人自我认知的边 界感探索产生了影响。尤其是可穿戴设备的嵌入，更加引发了对人与机器之间的界限到底在哪里，虚拟现实技术下什 么是人真实的存在等这些问题的思考。但是目前学界对可穿戴设备的研究较集中于技术材料与实际应用, 对于它与人 体本身概念的研究非常稀缺, 相关的实证研究不太充足, 而针对身体传播领域的研究在近些年来逐渐增多, 关于身体 这一命题的讨论声音慢慢兴起, 需要给予更多的关注进行发展。本文结合梅洛.庞蒂的身体现象学, 从现象学的角度思 考智能手环之类的可穿戴设备对知觉的身体、人的自我认知以及人体和机器的边界关系的影响, 以人为价值尺度, 探 讨可穿戴设备对人体边界感的探索与延伸。 
关键词: 智能媒介, 可穿戴设备, 智能手环, 梅洛·庞蒂

\section{1. 引言}

近年来, 人工智能日新月异的发展给人们的生产生活 带来了巨大变化, 其中智能媒介对人们生活领域的影响涉 及方方面面, 语音识别、人脸识别、算法分发等技术产生 了变革性影响。智能手环作为可穿戴设备之一, 慢慢在人 群中流行起来, 特别是中老年群体和运动群体, 它已成为 他们了解自己身体变化的主要媒介, 让他们可以跟自己的 身体进行更好地调节交互。智能手环通过感应器感知身体 细微变化, 具体成具体数据呈现于屏幕, 成为人的“智能 皮肤”, 展现人的动态和周围环境的变化。智能手环的使 用和普及是可穿戴设备嵌入人们身体生活的一大实践, 人 与机器之间的边界逐渐变得模糊起来, 人机交互的关系日 益复杂起来。当可穿戴设备逐渐进入人们生活, 对人自身 认知的影响、人与智能媒介的关系、人在虚拟技术下的真 实存在等, 这些问题都需要被仔细讨论。本文结合梅洛·庞 蒂的身体现象学理论知识, 对可穿戴设备对人自身身体边 界的探索延伸进行梳理和阐释。

\section{2. 可穿戴设备对人的延伸}

1975年Hamilton Watch推出了Pulsar计算器手表, 这款 产品的面世也意味着可穿戴设备在市场上的兴始[1], LED 的电子屏幕并且带有计算器功能的手表是当时产品上的 巨大突破。依托于互联网技术和科技高新技术的蓬勃发展, 可穿戴设备逐渐渗入医疗、教育、娱乐等各个生活领域, 为人们的生活带来了完全不同的体验。

\section{1. 目前关于可穿戴设备的研究}

目前对于智能可穿戴设备的研究主要集中于下面几 个方面:（1）可穿戴设备行业的发展前景与未来预期;

(2) 智能可穿戴设备其中的新型技术应用可能与提升强 化。学者王彰云2020年在其文章中设计提出以STM 32 为处 理器的智能手环以支持更强大的功能操作 [2]; (3) 可穿 戴设备的系统平台设计应用。学者陈春谋2019年设计采用 开源软件Android Studio, 为老年人健康监测系统的开发和 完善提供参考 [3]; (4) 可穿戴设备在相关领域的应用影 响。孙炎 戴启锐在2015年发表了探究可穿戴设备与医疗 健康产业关系的文章[4];（5）可穿戴设备在用户方面的 使用意愿和接受程度。

但是纵观前文这些对于可穿戴设备的研究, 很少有关 注度聚焦于它对于人的身体认知和感受变化, 而是更多的 在于技术提升和市场拓展方面。在人工智能、 $A R / V R$ 等技 术高速发展的背景下，人的虚拟现实和真实存在变成一个 非常大的问题, 人与机器的边界不再像以往这样清晰, 而 是变得模糊起来, 慢慢向着成人与机器共生的演变。那么 可穿戴设备对于的人的自我认识和边界意识到底会产生 什么样的影响, 是一个非常值得思考的问题。

\section{2. 可穿戴设备的种类功能及其对人的延伸}

目前可穿戴设备按照穿戴部分基本可分为头部穿戴 设备、手部穿戴设备、躯干穿戴设备以及下肢穿戴设备 4 种。[5]按照其功能也可进行划分, 远程操控、娱乐教育、 医疗健康、运动健身等等。

这些可穿戴设备的功能在这几年来通过技术的发展 也是非常多种多样, 心跳检测、运动计步、及时呼救等 等, 但是总的来说可以归纳为, 自我测量和外部沟通两 类功能。自我测量, 就是依靠传感器对对象的心跳、呼 吸、血压等生理机制进行测量, 然后通过显示技术进行 电子屏显化。这类主要集中于医疗健康和体育运动之中, 例如创业公司Empatica在14年推出的一款可通过检测皮 肤电追踪癫㾁患者的智能手环。而外部沟通功能主要是 将外界的温度、湿度、光度等环境变化放大, 让用户感 知, 同时结合互联网技术营造一个虚拟环境, 让用户可 以身临其境。谷歌在 2012 年就发布了一款谷歌眼镜 （Google Project Glass）用以“拓展现实”，用户可以佩戴 它利用虚拟界面进行拍照、电话、发送邮件等同智能手 机一样的操作。

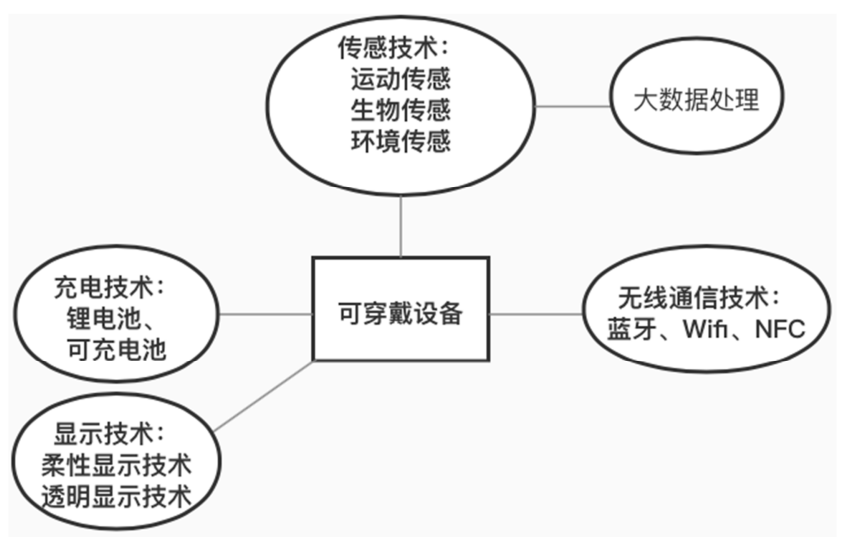

图1 可穿戴设备目前的使用技术[5]。

可穿戴设备自我测量和外部沟通这两个功能带来对 人的延伸体现在两个方面, 身体嵌入交互和触达范围扩大。 身体嵌入交互方面来看, 可穿戴设备佩戴于身上逐渐成为 身体的一部分, 可以代替人的部分器官工具的功能。2014 年巴西世界杯的开球仪式上, 一名名叫朱利亚诺.平托的 青年身着机器战甲, 靠着坚强的意志在脑机接口和机械外 骨的帮助下完成了开球, 令人震撼。其中的脑机接口技术 就是在人的大脑皮质灰层植入一块纳米级的芯片, 与外部 计算机等设备建立通路产生关联, 进行信息的交流来往。 [6]触达范围延伸则体现在可以与外界强大的通讯功能和 遥控功能, 可以作为人的身体部分延伸, 放大某种功能。 物联网的构建完善, 就是给可穿戴设备与更大的生活系统 连接提供了机会。 


\section{3. 智能手环}

智能手环是可穿戴设备在医疗健康管理领域中新兴 技术与信息结合的产品。智能手环的研究应用普及，可以 让人们对自身身体变化更加敏感, 在对身体数据的综合分 析后，可以更好预防相关疾病，进行健康管理。与此同时 智能手环还可以对周围环境进行实时监测, 甚至于与其他 用户进行无障碍的沟通交流，满足用户的社会性要求，更 好地与环境、社会相接洽。
智能手环的运作一般由五个部分共同完成, 它们分别 是, 手环 (带有感应器和采集器)、手机 (数据采集终端)、 $\operatorname{APP}$ (人机交互展示界面)、数据后台 (数据网络云储存)、 数据挖掘器（全网大数据挖掘中心）。当这五个部分共同 运作后，用户可以通过智能手环这个产品，获得巨大的使 用价值。[7]

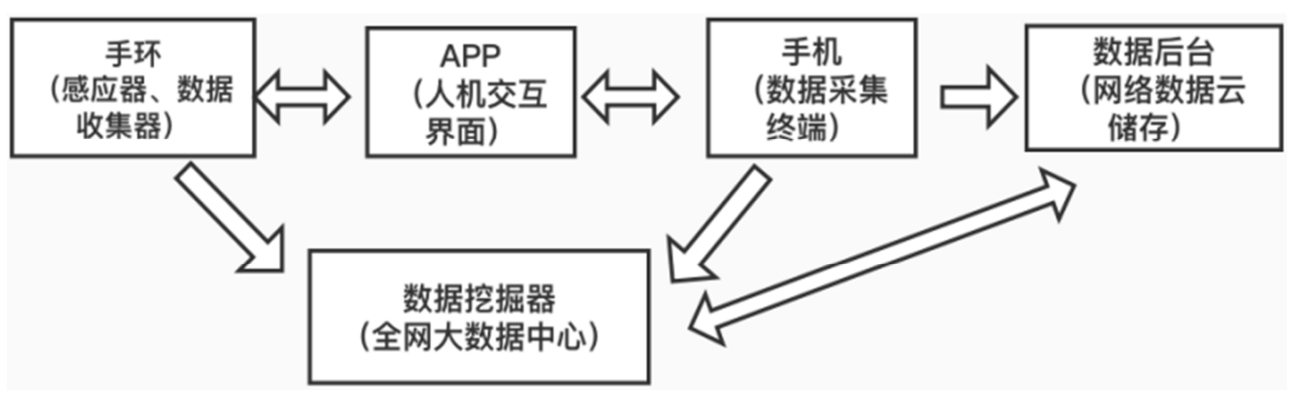

图2 智能手环运作链环。

\section{3. 知觉身体与时空延展}

从古希腊时代时，人们对于身体的认知一般停留在二 元对立的理论认知中, 主体-客体, 灵魂-肉体, 物质-意识。 一直到近现代, 随着生命科学、仿生学、心理学、哲学等 学科领域不断进步发展, 人们才逐渐改变这种二元对立的 思想，对“我”的概念不断深入。在这样的时代背景下，法 国学者梅洛·庞蒂在思考结合胡塞尔的现象学与笛卡尔的 二元论后, 建立了身体现象学这一身体哲学。在其著作《知 觉现象学》他提出了“身体图式”的概念, “身体图式是一 种表示我的身体在世界上的勋在方式”。[8]身体图式的概 念不再将身体和意识相对立分开, 而是将身体与时空运动 相结合，更加注重“此间”这一命题的理解。“只有打破传 统的对象化身体的思维模式, 把“现象身体”放到空间问题 的中心, 才能真正地理解与人的空间性存在相关的“现象 空间””[9]对我们如何理解现在智能技术带来的时空延展 对于身体认知的改变提供了非常有价值的思考。

\section{1. 知觉的身体与人的感知}

当我们描述一件眼前的物件时，我们总是下意识将对 方当成观察的客体，而忽视了我们自身也正处于这个“场” 当中, 看的事物正是这个“场”中所反映的现象, 正是被纠 缠于这个知觉场中我们才能作出这样的感知。我们现在知 道我们现在所默认看到的蓝色的天空、绿色的草地, 不过 是视觉机体对世界的反射, 而如果换一种视觉机制, 比如 狗或者猫的视觉系统, 那么对于这个世界的感知认同将会 完全不同。总的来说, 就是对于各种性质的感知是伴随着 外部运动一起带来的, 而不是完全固定不变的或者单一存 在的。这样来说, “眼见为实, 耳听我虚”这句话就是比较 荒谬的, 因为不论“眼见”还是“耳听”都是我的身体器官兴 奋反应, 它们是世界存在的一部分, 但是不意味着它们可 以设置世界可以被看见和被听见的范围。“在自在和自我 之间, 没有介于之间的东西, 因为即使我的感官有好几个,
但它们不是我本人, 只不过是物体。”[8]是因为我（Je） 将感知投射分散于被感知的事物上, 所以才会有这样的感 知经验解释。对于事物的感知并不是基于这个事物的本身, 而是一种意识的指向, 就像不论北京还是东京, 对于圆形 的感知是相同的。梅洛·庞蒂在书中竭力向我们阐释, 人 的身体知觉与感知体验都是建立在一个体验系统中, 相互 之间是牵引结合的，而不是支离破碎的。

\section{2. 时空运动与身体感知}

梅洛·庞蒂对于时间及时间观的思考源于现象学创始 人胡塞尔的结晶, 但是不仅仅是对胡塞尔的时间论进行了 继承, 他还作出了更进一步的反思, 提出了一些不同的观 点。“他充分肯定并借鉴胡塞尔现象学 内时间意识模式内 核一一意向性的意识流即体验流，体验流即时间流，时间 流是时间性的, 时间性即绝对主体性。”[10]但是在胡塞尔 对时间连续性的基础上，梅洛·庞蒂更近了一步，将时间 性与身体空间相结合, 阐释了身体层面上时间的连续一致 性。“梅洛-庞蒂通过聚焦“现”存的身体存在与世界之间的 知觉联系，赋予时间上的“当下’以优先权”[11]

对于身体的最初认知多数人大约就是建立在时空环 境的位置比对中, 比如, 我的手臂抬在胸前。形成对身体 各个部位的整体空间认知, 不是由于各个部位单纯的位移 结合或者各个部分的整体意识, 而是身体图式根据它们对 机体计划 (projets) 的价值基础上进行组合的结果。这里 非常有意思的就是对幻肢现象的讨论, 某些特殊情况下人 们失去了肢体的一部分, 但是他们在感觉上仍旧认为自己 没有失去肢体。在梅洛. 庞蒂他分析看来, 这种现象的出 现正是说明了, 身体并不是单纯的感知材料, 而同时是一 种心理层面的反映。

梅洛·庞蒂他将身体看作了客体所处环境中各个维度 的时间与空间下身体图式运动的综合感知, 认为“生存运 动不是靠把运动和视觉置于“我思...... 的支配之下, 而是 靠把运动和视觉引向一个“世界”的感觉间统一性, 才把运 
动和视觉联系在一起。”[8]正是通过与时空运动结合, 人 们才能从中获得有价值的东西, 找到生命意义。对于色盲、 神经功能失调等病症现象的举例分析, 梅洛·庞蒂同时证 明了身体图式对于运动分化能力的重要性。

\section{3. 具身化的身体与虚拟化的现实}

前文的赘述建立于可穿戴设备未出现的背景之下, 那 么在可穿戴设备日益普及的今天, 梅洛.庞蒂与他的身体 现象学是否依旧适用且具有参考价值呢? 答案当然是肯 定的。身体现象学对于人身体的讨论是超脱于以往二元论 中肉体的单一指向, 并不把身体等同于肉体, 而是将其看 作是身体图式与时空运动融合的产物。这恰恰与当今可穿 戴设备的身体嵌入以及功能延伸拓展带来的身体认知问 题相呼应, 当身体不再是我们往常所认为的肉体, 身体的 定义需要被重新赋予, 什么是身体, 身体与机器的界限在 哪里, 人机关系到底应该如何处理......这些问题需要被重 新思考。而身体现象学正是给我们提供了一个非常好的思 路, 来重新定义我们的身体。

\subsection{1. 可穿戴设备与具身性}

以往的身心二元论中, 人们将身体看作是装配意识的 容器, 并没有将身心看作是一体联动的。而梅洛. 庞蒂在 《知觉现象学》中首先提到了“具身性” (1'incarnation) 这 个概念, 具身性的概念破除了前面的身心二元论, 而建立 了身体-意识-世界动态交互的身体观念。“人的存在既非离 身的心智(disembodimentmind)也非复杂的机器, 其主体性 即在于作为活跃的生物（livingactive creatures）以人类身 体所特有的生理结构介入世界。”[12]所以说, 身心在参与 时空运动之中合一成为一整体而获得生命经验, 这正是人 的存在与复杂机器的本质区分。具身性需要讨论两个方面, 一是“嵌入性”, 二是“交互性”。当可穿戴设备嵌入身体时, 比如穿戴智能手环的后, 人手环作为与身体动态接轨的结 构算不算身体的一部分? 在身体现象学的理论概念下, 智 能手环作为身体化身的一部分, 参与时空运动中, 与身体 器官共同形成对自我、客体和他者的经验, 并赋予其特殊 的意义, 可以看做身体的构成, 因为身体的概念并不局限 于实体（entity）。可穿戴设备通过显示技术和感应器、 数据收集器等技术构建起人体与自我、环境的沟通的桥梁, 比如智能手环中的APP和屏幕界面就是人机交互关系节 电, 可以促进推动身体感觉和运动的效率。具身化包括这 些机器和人工产物融于我们身体中带来的体验。总而言之, 可穿戴设备完成了人的具身化。

\subsection{2. 可穿戴设备的“场”与人}

“场”是梅洛·庞蒂所讨论的一个概念, 但是他没有局限 一般物理意义上的在场或者缺席, 而是用一种全新的视角 来审视存在, 他认为的“场”是人们通过身体在原场域和知 觉域两者变动差异之上所获得的的能力 (puissance)。“外 在事物是否显现之场并非物理学意义上的可观场域, 而是 我先于一切科学认识的原始知觉的场域”[13]所以在这个 概念之上, 可穿戴设备所投射的虚拟场景不会因为其虚无 的存在, 而被排除在“场”之外, 它仍旧在身体现象学所讨 论的时空运动背景下。
智能手环所附带的远程遥控物体、通讯以及其他投射 虚拟场景功能, 虽然改变了客观环境的因素, 但是总的来 说还是没有动摇时空运动的本质，所以“场”的变化也正给 予人类更加广阔的时空运动能力, 可以有助于人类获得更 加丰富生命体验, 并不改变本质。

\section{4. 智能之下的忧虑}

可穿戴设备沈入人们生活, 人们裹挟在技术浪潮之下, 逐渐忘记了身体本身的意义。正如彼得斯在《对空言说》 中说的, “传播中的身体观念, 他在提出“在人类交流中人 体在多大程度上可以保持缺席”这个问题后总结了人类传 播观念中的身体问题史, 为讨论传播中身体问题提供了一 个非常好的历史语境” [12]传播中身体的问题是一个非常 值得思考的问题。“智能媒介对传播本身产生的变革效果, 会扩展为深入社会文化层面的媒介效应, 更进一步的现代 化意味着人类将面临感觉丰富性和主体性被进一步剥夺 的风险。”[14]关注可穿戴设备等智能媒介在给人带来的便 捷益处之外, 所暗含的可能风险, 思考人类与媒介, 媒介 与社会等更深层次的关系。

\section{1. 可穿戴设备与身体依赖}

目前争议最大的可穿戴设备非智能手机莫属, 手机依 赖症成为现代人的典型“病症”之一, 多数人手机不离身, 甚至有人更加严重一旦失去了手机就会坐立不安产生室 息感和空虚感。可穿戴设备如果长期后, 也许会代替延伸 身体的一部分功能, 进而在心理上成为身体必不可少的一 部分, 容易产生依赖感。

\section{2. 可穿戴设备与身体隐私泄露}

可穿戴设备一般带有数据采集器, 将所采纳的身体数 据传送到后台的大数据中心进行处理, 这可能会带来一个 问题, 那就是身体隐私数据的泄露。在享受智能功能的同 时, 也不能忘记身体还有个人隐私的从属。“可穿戴设备 的数据安全与隐私保护问题的研究大多集中在某一层面, 特别是技术层面的算法及网络安全等, 对管理、伦理等方 面隐私问题讨论较少, 欠缺整体性和综合性。”[15]

\section{5. 结语}

诸如智能手环之类的可穿戴设备的使用, 不仅在物理 层面上延伸嵌入了人体之中, 而且在意识层面也逐渐改变 了人们对身体边界的认知。以设备界面作为人机交互的中 介, 凭借传感器、大数据处理、无线通信、信息显示等技 术, 可穿戴设备实现自我测量和外部沟通两个功能, 使得 身体与意识、世界联结进行时空互动。

作为高新技术相结合的产物, 智能手环之类的可穿戴 设备确实延伸了人们的感知体验, 丰富拓展了人的时空运 动体验, 但是并没有破坏人的身体边界, 反而探索延伸了 对身体的定义。梅洛·庞蒂的身体现象学理论解答了智能 媒介使用下, 自为、自在、他者、意义四者该如何融合自 洽。可穿戴设备在嵌入身体后, 参与到身体的时空运动之 
中, 推动身体感觉的进化和转化, 提升了运动的效率, 帮 助人们完成了具身化。

在智能媒介逐渐普及的今天, 我们需要注意可穿戴设 备的使用可能会带来依赖症、隐私泄露等问题, 给我们的 生活带来一定的麻烦。但是总的来说, 在注意规避它的不 良影响下使用可穿戴设备, 对于人来说还是会带来全新的 生命体验, 是非常有益的。

\section{参考文献}

[1] 刘舒佳.可穿戴设备发展史[J].信息方略, 2014，(10）：17。

[2] 王彰云.基于STM32智能手环控制系统设计与实现 [J]. 湖北 农机化，2020,(17): 13-20。

[3] 陈春谋.基于Android系统的智能手环健康检测系统关键技 术 $[J]$.微型电脑应用，2019，（35）：99-101。

[4] 孙炎, 戴启锐.可穿戴设备与医疗健康产业关系研究及发展 趋势分析 $[\mathrm{J}]$.中国数字医学, 2015, (08)：25-28。

[5] 蒋小梅, 张俊然, 陈富琴, 赵斌.可穿戴式设备分类及其相 关技术进展 $[\mathrm{J}]$. 生物医学工程学杂志, 2016, (01) : 42-48。

[6] 张田勘.世界杯上的脑控机械腿 [J]. 北方人, 2014,(09):55-56。
[7] 许丽娟, 欧阳伟坚, 刘裕, 李美玲.大数据与智能手环 $[\mathrm{J}]$. 智能城市，2016，2（02）：29-33。

[8] 莫里斯·梅洛-庞蒂. 知觉现象学 [M].姜志辉, 译. 北京: 商务 印书馆, 2001。

[9] 庞西院.梅洛-庞蒂的现象空间：身体、知觉与体验 $[J]$.长沙 理工大学学报（社会科学版），2014，29（05）:37-41。

[10] 王全宁.从梅洛一庞蒂的身体图式概念出发重新认识人 自 己-— 兼论价值以人自身为尺度 $[\mathrm{J}]$.湖北大学学报 (哲学 社会科学版），2013，（02）：1-9。

[11] 王亚娟.梅洛-庞蒂与海德格尔之间“缺失的对话”[J].哲学动 态, 2014, (10)：43-52。

[12] 刘海龙, 束开荣. 具身性与传播研究的身体观念一一知觉 现象学与认知科学的视角 [J]. 兰州大学学报 (社会科学版), 2019 (047) ，002: 80-89。

[13] 庄元.手.持存.现在一一论读解《知觉现象学》的一种可能 视角 $[\mathrm{J}]$.理论月刊, 2018, (12)：63-68。

[14] 胡万鹏. 后现代视域下对智能媒介的批判与反思 $[\mathrm{J}]$. 青年记 者, 2018，(32)：12-13。

[15] 何晓林, 钱庆, 吴思竹, 张泽, 孙小康. 健康医疗可穿戴 设备数据安全与隐私研究进展 $[\mathrm{J}]$. 中华医学图书情报杂志, 2016, 25 (10)：32-37。 